University of New Mexico

UNM Digital Repository

3-1-2004

\title{
Effects of body size and temperature on population growth
}

Van M. Savage

James F. Gillooly

James H. Brown

Geoffrey B. West

Eric Charnov

Follow this and additional works at: https://digitalrepository.unm.edu/biol_fsp

Part of the Biology Commons

\section{Recommended Citation}

Savage, V.M., J.F. Gillooly, J.H. Brown, G.B. West and E.L. Charnov. 2004. Effects of body size and temperature on population growth. American Naturalist 163:429-441

This Article is brought to you for free and open access by the Academic Department Resources at UNM Digital Repository. It has been accepted for inclusion in Biology Faculty \& Staff Publications by an authorized administrator of UNM Digital Repository. For more information, please contact disc@unm.edu. 


\title{
Effects of Body Size and Temperature on Population Growth
}

\author{
Van M. Savage, ${ }^{1,2, *}$ James F. Gillooly, ${ }^{3}$ James H. Brown, ${ }^{1,3}$ Geoffrey B. West, ${ }^{1,2}$ and Eric L. Charnov ${ }^{3}$
}

1. Santa Fe Institute, Santa Fe, New Mexico 87501;

2. Theoretical Division, Los Alamos National Laboratory, Los Alamos, New Mexico 87545;

3. Department of Biology, University of New Mexico, Albuquerque, New Mexico 87131

Submitted August 20, 2002; Accepted August 20, 2003; Electronically published March 9, 2004

Online enhancements: appendix, table, literature cited.

ABstract: For at least 200 years, since the time of Malthus, population growth has been recognized as providing a critical link between the performance of individual organisms and the ecology and evolution of species. We present a theory that shows how the intrinsic rate of exponential population growth, $r_{\max }$, and the carrying capacity, $K$, depend on individual metabolic rate and resource supply rate. To do this, we construct equations for the metabolic rates of entire populations by summing over individuals, and then we combine these population-level equations with Malthusian growth. Thus, the theory makes explicit the relationship between rates of resource supply in the environment and rates of production of new biomass and individuals. These individual-level and population-level processes are inextricably linked because metabolism sets both the demand for environmental resources and the resource allocation to survival, growth, and reproduction. We use the theory to make explicit how and why $r_{\max }$ exhibits its characteristic dependence on body size and temperature. Data for aerobic eukaryotes, including algae, protists, insects, zooplankton, fishes, and mammals, support these predicted scalings for $r_{\max }$. The metabolic flux of energy and materials also dictates that the carrying capacity or equilibrium density of populations should decrease with increasing body size and increasing temperature. Finally, we argue that body mass and body temperature, through their effects on metabolic rate, can explain most of the variation in fecundity and mortality rates. Data for marine fishes in the field support these predictions for instantaneous rates of mortality. This theory links the rates of metabolism and resource use of individuals to life-history attributes and population dynamics for a broad assortment of organisms, from unicellular organisms to mammals.

* Corresponding author. Address for correspondence: Santa Fe Institute, 1399 Hyde Park Road, Santa Fe, New Mexico 87501; e-mail: van@santafe.edu.

Am. Nat. 2004. Vol. 163, pp. 429-441. (c) 2004 by The University of Chicago. 0003-0147/2004/16303-20308\$15.00. All rights reserved.
Keywords: allometric scaling, population growth, body size, temperature, population dynamics, $r_{\max }$, carrying capacity.

Perhaps the most long-standing and well-accepted "law" of ecology is the inherent capacity of all organisms for exponential population growth. In An Essay on the Principle of Population, Thomas Malthus (1798) noted that all populations had the potential to increase geometrically" and that this potential would be realized under favorable conditions where resources are abundant and enemies are absent. This capacity was a cornerstone of Darwin's theory of evolution by natural selection, and it is central to a large body of theory in contemporary ecology and evolution. For example, Turchin (2001) considers Malthusian or exponential population growth to be one of the few universal laws of ecology.

This law is usually written as

$$
N(t)=N(0) e^{r t} \text { or } \frac{d N}{d t}=r N,
$$

where the size of the population, $N$, at some time, $t$, is a function of initial population size, $N(0)$, and the specific rate of exponential population growth, $r$. Depending on the environmental conditions, $r$ can be negative, positive, or 0 . However, there are two choices of $r$, corresponding to two special conditions, that are the most natural choices for comparisons across populations and are the most well studied in the ecological literature, both empirically and theoretically. The first of these, $r=r_{\max }$, represents the inherent capacity of a population to reproduce and is thus the maximum exponential growth rate when resources are not limiting. The second choice for comparisons across populations is the steady state situation, $r=0$, where the total number of individuals in the population does not change with time. For this case, the number of organisms at equilibrium is referred to as the carrying capacity, $K=N(r=0)$, of the environment.

Ecologists and evolutionary biologists have long recognized that $r$ and $K$ vary enormously among different taxonomic or functional groups of organisms and with changing environmental conditions. Microbiologists have attempted to understand this variation in terms of first 
principles of biology, physics, and chemistry. Their contributions have had only limited influence in ecology, however, perhaps because ecologists have had difficulty relating population growth of microbes in culture to population growth of plants and animals in the field or laboratory. The link is provided by metabolism, the complex of biochemical reactions that governs the flow of energy and transformation of materials in organisms. The metabolic rate is the most fundamental biological rate, and it determines the rates of most other organic processes. In particular, the metabolic rates of individual organisms determine the vital rates of survival, growth, and reproduction, and these in turn determine the growth rates of populations.

It has long been known that metabolic rates vary in predictable ways with body size and temperature. Recent progress in theoretically characterizing the joint effects of size and temperature on metabolic rate (Gillooly et al. 2001, 2002) provides the basis for relating metabolic rates of individual organisms to an increasing number of ecological and evolutionary processes (e.g., Allen et al. 2002; Brown et al. 2003). Here, we show that the body size and body temperature dependence of growth rates of populations is a direct consequence of the body size and body temperature dependence of the metabolic rates of individual organisms. Therefore, size and temperature can explain much of the variation in $r_{\max }$ and $K$ among different kinds of organisms and environments.

We emphasize that many ecologists have recognized the body size and temperature dependence of population growth rate. For example, it has long been known that $r_{\max }$ scales with the $-1 / 4$ power of body mass (Fenchel 1973; Southwood et al. 1974; Blueweiss et al. 1978; Hennemann 1983; Peters 1983) and that carrying capacity, $K$, scales with the $-3 / 4$ power of body mass (Damuth 1987; Belgrano et al. 2002). Although the temperature dependence of $r_{\max }$ has also been studied extensively, especially in microbes and insects (Monod 1942; Birch 1948; Dean and Hinshelwood 1966; Droop 1968; Eppley 1972), the temperature dependence of $K$ has received surprisingly little attention. Despite these empirical findings, a general theory that explains these mass and temperature scalings for both maximal and steady state population growth across a wide range of species is lacking. Moreover, with few exceptions (Peppin 1991), the combined effects of mass and temperature have not been considered; for example, studies have investigated just the body mass dependence for larger organisms such as fishes, amphibians, and reptiles or just the temperature dependence for smaller organisms such as microbes and insects.

Here, we begin by developing a general theory for population growth that relates any value of $r$ to individual metabolic rate, $B$. Then we focus on the special cases, $r=0$ and $r=r_{\max }$ in order to derive scalings for population growth and life-history parameters. Life-history strategies for various organisms determine which parameters are the most relevant to study. Therefore, we develop special applications of this theory on the basis of what fraction of an organism's life span is spent reproducing, which we divide according to nonoverlapping and overlapping generations, and on the basis of whether an organism's ontogenetic growth is determinate or indeterminate. Because previous work connects metabolic rate to body mass and body temperature, we can use this framework to predict how population growth and life-history parameters depend jointly on both body mass and body temperature. Using published data, we then evaluate these predictions and find support for our theory. Finally, we comment on some of the implications of this theory for ecology and future directions of research.

\section{Theory Relating Population Growth Rate to Metabolic Rate, Body Mass, and Temperature}

Physiologists define metabolic rate to be the rate at which organisms transform energy through biochemical processes. West et al. (1997) and Gillooly et al. (2001) showed that the mass and temperature dependence of the whole organism metabolic rate, $B(m, T)$, for an individual scales as

$$
B(m, T)=B_{0} e^{-E / k T} m^{3 / 4},
$$

where $E$ is the average activation energy of rate-limiting biochemical metabolic reactions, $k$ is Boltzmann's constant, $T$ is absolute temperature, $B_{0}$ is a taxon-dependent and metabolic-state-dependent normalization constant, and $m$ is the body mass of an individual. The mass dependence in equation (2) is a consequence of the scaling of resource supply networks and exchange surfaces in branching hierarchical networks (West et al. 1997; see also Banavar et al. 1999, 2002). As for the temperature dependence in equation (2), rates of chemical reactions, including biochemical reactions of metabolism in the body, scale with temperature according to $e^{-E / k T}$, known as the Boltzmann factor in physics and the Arrhenius-van't Hoff expression in ecology and biochemistry. Therefore, the temperature in equation (2) is the temperature at which the biochemical reactions are occurring, which is the same as the body temperature of the organism. For ectotherms, this is very nearly equal to the environmental temperature, but for endotherms, it is high $\left(35^{\circ}-40^{\circ} \mathrm{C}\right)$ and virtually independent of environmental temperature. Gillooly et al. (2001) show empirically that this mass and temperature dependence explains much of the variation in the metabolic rate within groups of animals, plants, and microbes. 
Different taxonomic or functional groups may differ by as much as a factor of 20 .

To a very good approximation, equation (2) applies to different levels of metabolic activity (Peters 1983), but just as for $r$, a standard must be chosen to compare across species. This choice partially determines $B_{0}$. The most common choices are basal metabolic rate, the metabolic rate of a fasting, inactive individual; field metabolic rate, the metabolic rate of a free-living individual in its environment; and maximal metabolic rate, the metabolic rate of an individual at maximum aerobic activity. The rates at which organisms acquire energetic and material resources from the environment, transform them in biochemical reactions, and allocate them to maintenance (survival), growth, and reproduction are inextricably linked to metabolic rate. In this article, we are interested in how an individual allocates its metabolic rate between the power it uses for maintenance and the power it uses to create new organisms. The metabolic rate that is most relevant in this article is closely akin to field metabolic rate.

We begin by showing the relationship between the metabolic rate of a single individual and the average metabolic rate for an individual in a population. A population growing exponentially in a constant environment at a fixed rate, $r$, will reach a stable age and size distribution, so the average value of mass for an individual in the population, $M$, becomes time independent. Therefore, if we replace the mass term for an individual, $m$, by $M$, we can define the time-independent quantity, $B(M, T)=B_{0} e^{-E / k T} M^{3 / 4}$, which is an excellent approximation to the average metabolic rate of an individual (Savage 2004).

Next, we examine how organisms allocate their metabolic power to maintenance, replacement, and population growth. If individuals are just sustaining themselves and not reproducing, the metabolic rate of the population can be obtained by multiplying the average maintenance metabolic rate of an individual, $B(M, T)$, by the number of individuals in the population at time $t, N(M, T, t) B(M, T)$. If the population is at a steady state, $r=0$ and $N=K$, and each individual is on average just replacing itself, then some energy and power must be allocated to creating new individuals. Defining $E(M)$ to be the energy to produce a new individual (this quantity is independent of temperature, which affects only the rate of ontogenetic growth), it takes $N(M, T, t) E(M)$ amount of energy to replace the entire population. Because on average $N(M, T, t)$ deaths will occur over a time period equal to the average life span, $S(M, T)$, all individuals will be replaced during the time interval of a single life span, so the power needed to keep the size of the population at steady state is given by $N(M, T, t) E(M) / S(M, T)$. Finally, if the population is not just maintaining its numbers but increasing them, the rate of creating new individuals beyond replacement is $d N(M, T, t) / d t$, so $E(M) d N(M, T, t) / d t$ is the power given to population growth. Even when the population is decreasing in size, $E(M) d N(M, T, t) / d t$ measures how quickly the metabolic rate of the entire population is decreasing as a result of a net loss of individuals and must be included as part of the total metabolic rate. Consequently, by summing the power required for maintenance, replacement, and net new individuals, we express the total metabolic rate of the population as

$$
\begin{aligned}
B_{\mathrm{POP}}(M, T, t)= & N(M, T, t)\left[B(M, T, t)+\frac{E(M)}{S(M, T)}\right] \\
& +E(M) \frac{d N(M, T, t)}{d t} .
\end{aligned}
$$

To review, after expansion, the first term in equation (3), $N(M, T, t) B(M, T)$, is the power required for maintenance; the second term, $N(M, T, t) E(M) / S(M, T)$, is for replacement (i.e., production of one offspring per individual) at zero population growth; and the third term, $E(M) d N(M, T, t) / d t$, is for production of offspring in addition to replacement. Although $M$ and the metabolic rate per individual for maintenance, $B(M, T)$, are time independent, the metabolic rate of the entire population, $B_{\mathrm{POP}}(M, T, t)$, can vary with time because the number of individuals in the population, $N(M, T, t)$, often varies with time. From equation (3), the total mass-specific metabolic rate for an individual averaged over the entire population is given by

$$
\begin{aligned}
\bar{B}_{\mathrm{POP}}(M, T, t) & \equiv \frac{B_{\mathrm{POP}}(M, T, t)}{N(M, T, t) M} \\
& =\bar{B}(M, T)+\frac{E(M)}{M S(M, T)}+\frac{E(M)}{M} r
\end{aligned}
$$

where $\bar{B}(M, T) \equiv(B(M, T) / M)$ is the mass-specific metabolic rate for maintenance of an individual, and we have used equation (1) to replace $(1 / N)(d N / d t)$ with $r$. Equation (4) is a general equation that applies to any value of $r$ and is valid for any conditions that do not violate the assumption of a stable age and size distribution. For the special case of a nongrowing population, $(d N / d t)=r=$ 0 , the last term in equation (4) is 0 , and the average massspecific metabolic rate of an individual that is just replacing itself is $\bar{B}_{\mathrm{REP}}(M, T)=\bar{B}(M, T)+(E(M) / M S(M, T))$. Therefore, the average mass-specific metabolic rate of an individual in a maximally increasing population, $\bar{B}_{\text {MAX }}(M, T)$, must exceed that of an individual in a stable population, $\bar{B}_{\mathrm{REP}}(M, T)$, which in turn must exceed the mass-specific metabolic rate of an individual that is main- 
taining itself without growing or reproducing, $\bar{B}(M, T)$; that is, $\bar{B}_{\mathrm{MAX}}(M, T)>\bar{B}_{\mathrm{REP}}(M, T)>\bar{B}(M, T)$.

We next consider how size and temperature affect the rate of energy allocation to production of new individuals. We assume that the energy required to produce a new individual is linearly proportional to its mass, $E(M)=$ $E_{0} M$, where $E_{0}$ is a normalization constant. Because this cancels the explicit $M$ dependence in equation (4) and because life span increases with increasing body size and decreasing temperature, the mass-specific energy required for an individual to replace itself scales inversely with life span. Empirically, life span scales inversely with the massspecific metabolic rate, so

$$
\frac{E(M)}{M S(M, T)}=\frac{E_{0}}{S(M, T)}=\frac{E_{0}}{S_{0}} \bar{B}(M, T),
$$

where $S_{0}$ is a constant given by $S(M, T)=\left(S_{0} / \bar{B}(M, T)\right)$ (Gillooly et al. 2001). Thus, $\bar{B}_{\mathrm{REP}}(M, T)=[1+$ $\left.\left(E_{0} / S_{0}\right)\right] \bar{B}(M, T)$ and

$$
\begin{aligned}
\bar{B}_{\mathrm{POP}}(M, T, t) & =\bar{B}_{\mathrm{REP}}(M, T)+E_{0} r \\
& =\left(1+\frac{E_{0}}{S_{0}}\right) \bar{B}(M, T)+E_{0} r
\end{aligned}
$$

which relates the average total mass-specific metabolic requirements of an individual to its requirements for maintenance and reproduction.

Now we are in a position to determine the mass and temperature dependence of $r$ and make empirically testable predictions. We do this using the Euler equation,

$$
1=\int_{0}^{\infty} d x e^{-r x} L(x) b(x),
$$

where the integral is performed over all possible ages, $x$, of individuals; $b(x)$ is the average fecundity at age $x$; and $L(x)$ is the percentage of the population that has survived from birth to age $x$ (Charnov 1993). In appendix A in the online edition of the American Naturalist, we show that under the assumption that $L(x) b(x)$ is a function that is sharply peaked around the average age at which a female gives birth, known as the generation time, $G$, it follows that $r$ is inversely related to $G$ (May 1976). A simple argument relates these quantities to the average number of offspring per individual per generation, $R_{0}$. Using equation (1) to express $R_{0}$ gives $r G=\ln \left(R_{0}\right)$. Because the factor $\ln \left(R_{0}\right)$ is slowly varying, it can be considered constant, so the mass and temperature dependence of $r$ must vary inversely to that of $G$.
Gillooly et al. (2002) previously derived a relation for time to first reproduction, $\alpha$, in terms of mass and temperature

$$
\alpha \propto M^{1 / 4} e^{E / k T} .
$$

This theoretically predicted relation is supported by empirical data $\left(r^{2}=0.74\right.$ for zooplankton; Gillooly et al. 2002). As will be shown later, $G$ scales in the same way as does $\alpha$, so $G \propto M^{1 / 4} e^{E / k T} \propto(1 /(\bar{B}(M, T)))$, which is consistent with the close correlation between generation time and time to first reproduction. Therefore,

$$
r=\frac{\ln R_{0}}{G} \approx r_{1} M^{-1 / 4} e^{-E / k T}=\frac{r_{1}}{B_{0}} \bar{B}(M, T),
$$

where $r_{1}$ is a taxon- and environment-dependent normalization constant. Note that for the special case of a nongrowing population, we have $r_{1}=0$, and $r$ is independent of mass and temperature.

Combining equations (5) and (8) gives

$$
\bar{B}_{\mathrm{POP}}(M, T, t)=r\left[\left(1+\frac{E_{0}}{S_{0}}\right) \frac{B_{0}}{r_{1}}+E_{0}\right] .
$$

This equation makes explicit the energetic constraints of metabolism on population growth. That is, $r$ determines the rate at which the population consumes energy resources, or conversely, the rate of resource supply limits the rate of population growth:

$$
r=\bar{B}_{\mathrm{POP}}(M, T, t)\left[\left(1+\frac{E_{0}}{S_{0}}\right) \frac{B_{0}}{r_{1}}+E_{0}\right]^{-1} .
$$

Recall that $E_{0}, S_{0}, B_{0}$, and $r_{1}$ are independent of $M$ and $T$, so the scaling of $r$ reflects that of the average mass-specific metabolic rate of an individual. Provided that the assumption of a stable age and size distribution and the approximation implied by equation (8) (see app. A) are valid, equations (3)-(5) and (9)-(10) apply to all values of $r$. For purposes of comparison and analysis of data, we now characterize the two special cases previously discussed, $r=0$ and $r=r_{\max }$.

\section{Steady State Populations $(r=0)$ : Body Size, Temperature, and the Energy Equivalence Rule}

Of considerable interest in ecology is the special case of a nongrowing population, where $(d N / d t)=r=0$, and there is a steady state between the rate of limiting resource supply in the environment and the rate of resource use 
by the population. Defining the rate of resource supply as $P$, the equilibrium population size or carrying capacity is reached, $N=K$, when $(d N / d t)=r=0$ and $B_{\mathrm{POP}}=P$. Consequently, using $(E(M) / S(M, T))=\left(E_{0} / S_{0}\right) B(M, T)$, equation (3) becomes

$$
P=\left(1+\frac{E_{0}}{S_{0}}\right) K(M, T) B(M, T) .
$$

For the special case where $P$ is constant, independent of $M$ and $T$, the scalings for $K(M, T)$ and $B(M, T)$ must balance so that $K(M, T) \propto B^{-1}(M, T)$. Because whole organism metabolic rate, $B(M, T)$, scales as $M^{3 / 4} e^{-E / k T}$, it follows that

$$
K(M, T) \propto e^{E / k T} M^{-3 / 4} .
$$

This predicts that for a fixed supply of resources, $P$, carrying capacity or equilibrium population size, $K(M, T)$, should decrease with increasing body size as $M^{-3 / 4}$ and also decrease with increasing temperature as $e^{E / k T}$. Because equation (12) assumes that $P$ does not depend on $M$ or $T$, it applies to species living in separate environments with identical resource supply rates or to coexisting species that have identical rates of resource use in the same environment; that is, some constant fraction of $P$ is used by each species. Consequently, equation (12) explains the $M^{-3 / 4}$ trade-off between size and abundance shown by plants of different sizes growing under near-constant conditions, that is, similar values of $P$ (Enquist et al. 1998). These equations do not explain how species apportion energy resources and power, $P$, or balance population abundances in a shared environment. However, if we assume the energy equivalence of species resource use in natural populations described by Damuth (1987), then equation (12) follows from our theory. Hence, equation (12) determines the mass and temperature dependence of carrying capacity in or across ecosystems where the total population of each species uses approximately the same amount of power. This body size dependence agrees with the previous findings of Damuth (1987; see also Belgrano et al. 2002). The Boltzmann temperature dependence is of at least equal importance. It agrees with Allen et al.'s (2002) findings for ectotherms across environmental gradients of latitude and temperature. The negative relationship between carrying capacity and temperature may seem counterintuitive, but it is a direct consequence of the fact that when operating at a higher temperature, each individual uses resources at a higher rate because of its elevated metabolic rate. Inserting the temperature dependence of $P$ into equation (11) should provide an especially useful method for making comparisons across latitudes, elevations (terrestrial), or depths (aquatic).

Equation (11) can also be used to place an upper bound on the value of $K(M, T)$ for each species. The power used by a given species cannot exceed the total power available in the environment, $P_{\mathrm{TOT}}$, so equation (11) dictates that

$$
\begin{aligned}
K(M, T) & \leq \frac{P_{\mathrm{TOT}}}{\left[1+\left(E_{0} / S_{0}\right)\right] B(M, T)} \\
& =\frac{P_{\mathrm{TOT}}}{\left[1+\left(E_{0} / S_{0}\right)\right] B_{0}} M^{-3 / 4} e^{E / k T} .
\end{aligned}
$$

Thus, because $P_{\text {TOT }}$ does not depend on $M$ or $T$, in a plot of $\log \left(K(M, T) e^{-E / k T}\right)$ versus $\log M$, all of the data must fall below a maximal line with a slope of $-3 / 4$ and an intercept given by $\log \left[\left(P_{\mathrm{TOT}} / B_{0}\right)\left(1+E_{0} / S_{0}\right)^{-1}\right]$. If carrying capacity, $K(M, T)$, scales with mass according to a power law that extends over an unbounded mass range, then equation (13) requires that the power law have a slope of $-3 / 4$; that is, $K(M, T) \propto M^{-3 / 4}$. If $K(M, T)$ deviated from a $-3 / 4$ power law, the two lines would not be parallel, and the line for the temperature-corrected carrying capacity would necessarily exceed the maximal line for certain masses, thereby violating equation (13). In actuality, the mass range is not unbounded, although mammals do span a very wide mass range, covering eight orders of magnitude. Moreover, values for the temperaturecorrected carrying capacity will probably be within two to three orders of magnitude of the upper bound in equation (13), corresponding to a separation of two to three units between the power-law line for temperature-corrected carrying capacity and the maximal line. Because these lines have a small separation of two to three units in the logarithm of temperature-corrected carrying capacity ( $Y$-axis) compared with the range of eight units in the logarithm of mass ( $X$-axis), the slope of the line for the temperaturecorrected carrying capacity must be close to $-3 / 4$, or the maximal line will be crossed, and equation (13) will be violated. Consequently, if carrying capacity does follow a power law for mammals, we have argued on very general grounds that the exponent for the mass must be close to $-3 / 4$.

\section{Theory for Nonoverlapping Generations with $r=r_{\text {max }}$ : Case 1}

Next, we consider populations where individuals reproduce during only a small fraction of their lifetime, hereafter referred to as discrete or nonoverlapping generations. One example is given by unicellular organisms, which reproduce by fission. Other examples are given by multicellular organisms, such as many insects and zooplankton, which 
spend only a brief period of their lives reproducing. For such organisms, $L(x)$ and $b(x)$ are 0 except at $x=\alpha=$ $G$. Equation (6) can now be solved to give $r_{\max }=$ $\ln [L(G) b(G)] / G$. Note that $\ln [L(G) b(G)]$ is a slowly varying function, so the mass and temperature dependence of $r_{\max }$ is dominated by the variation in $G$, which agrees with the approximation in appendix A. For realistic values of $L(G) b(G)$ ranging from 3 to $30, \ln [L(G) b(G)]$ ranges from only 1 to 3 . Thus, equation (8) is a good approximation to the solution, with $r_{1}=\ln [L(G) b(G)] / C$, where $C$ is a proportionality constant related to ontogenetic growth rate (Gillooly et al. 2002). In this case, the equations are easy to solve, and the approximations and predictions should be quite accurate.

\section{Theory of Overlapping Generations with $r=r_{\max }$ : Case 2}

Here, we consider organisms that reproduce over a significant fraction of their lifetimes so that several generations can be reproducing at the same time, hereafter referred to as overlapping generations. First, we consider the simpler case of determinate ontogenetic growth (e.g., birds and mammals), where growth ceases at the age of maturity, $\alpha$. Then we argue that the theory is robust enough also to describe indeterminate ontogenetic growth (e.g., fishes and most plants). For these organisms, the approximation used in appendix A does not apply because populations are age or size structured. Therefore, we use a different method for solving Euler's equation and in the process derive scaling laws for $b(x)$ and $L(x)$ (Charnov 1993).

We assume that this case is described by the production of offspring $(b)$ and the instantaneous mortality rate $(Z)$ both occurring at a constant rate. With $b(x)=0$ for $x<$ $\alpha$ and $b(x)=b(\alpha)$ and $L(x)=L(\alpha) e^{-Z(x-\alpha)}$ for $x>\alpha$, equation (6) can be written as

$$
1=b(\alpha) L(\alpha) e^{Z \alpha} \int_{\alpha}^{\infty} d x e^{-\left(Z+r_{\max }\right) x} .
$$

Integrating equation (14) and rearranging terms gives $\left(Z+r_{\max }\right) e^{r_{\max } \alpha}-b(\alpha) L(\alpha)=0$. In this expression, $L(\alpha)$ can be written as $L(\alpha)=e^{-\int_{0} Z(x) d x}=e^{-\bar{Z} \alpha}$, where $\bar{Z}$ is an average of $Z$ over the immature period of ontogenetic growth (Charnov 1993). Hence, equation (14) becomes

$$
\begin{aligned}
\left(Z+r_{\max }\right) e^{r_{\max } \alpha}-b(\alpha) e^{-\bar{Z} \alpha} & =0 \text { or } \\
\left(\bar{Z}+r_{\max }\right) \alpha & =-\ln \left(\frac{Z+r_{\max }}{b(\alpha)}\right) .
\end{aligned}
$$

The exponent in the first term must be dimensionless, so $r_{\max }$ has the dimensions of $1 / \alpha$. Because $e^{r_{\max } \alpha}$ increases rapidly with $\alpha$ and $e^{-\bar{Z} \alpha}$ decreases rapidly with $\alpha$, it appears as if equation (15) cannot be satisfied for all values of $\alpha$. However, equation (15) is always satisfied if $r_{\max }$ and $\bar{Z}$ both vary inversely with $\alpha$. These relations keep the exponentials fixed and the two terms in balance. Consequently, $r_{\max } \propto \bar{Z} \propto 1 / \alpha$, which suggests $\alpha \propto G$ as previously stated. Moreover, by a similar analysis, equation (15) shows that it is consistent for the coefficients $Z$ and $b$ to be proportional to $r_{\max }$ and, thus, that $Z, b$, and $\bar{Z}$ are all inversely related to $\alpha$ as given by equation (7). We propose that these are the scalings for this case, so

$$
Z(\alpha) \propto \bar{Z}(\alpha) \propto b(\alpha) \propto M^{-1 / 4} e^{-E / k T} .
$$

So, mortality rate is predicted to have the same mass and temperature dependence as $r_{\max }$ because both of these rates are determined by and scale the same as mass-specific metabolic rate.

Even though the above analysis is for organisms with determinate ontogenetic growth, the scaling relations may be robust enough to apply to a broad range of organisms including those with indeterminate ontogenetic growth (e.g., fishes). Below, we provide empirical evidence that for indeterminate growers, $Z$ does indeed scale according to equation (16) and that $r_{\max }$ scales like equation (8).

\section{Data and Methods}

The above predictions were evaluated by compiling relevant data from the literature. In the following analyses, we de-emphasized evaluating the mass dependence of $r_{\max }$ because the predicted $M^{-1 / 4}$ scaling has been well documented by previous studies (e.g., Fenchel 1973; Southwood et al. 1974; Blueweiss et al. 1978; Henneman 1983; Peters 1983).

\section{$r_{\max }:$ Laboratory Data for Invertebrates}

Data on intrinsic rate of increase, $r_{\max }$, for insects, zooplankton, algae, and protists, were compiled from laboratory studies where the organisms were incubated at constant temperatures ranging from $5^{\circ}-35^{\circ} \mathrm{C}$. Figure 1 illustrates the temperature dependence of population growth. Each species has a minimum temperature below which population growth cannot occur, an optimal temperature at which maximal growth occurs, and a very narrow temperature range above the optimum where population growth rates decrease rapidly. It is only in the "biologically relevant" temperature range, between the lower threshold and the optimum, that metabolic rates and population growth rates have exponential temperature 


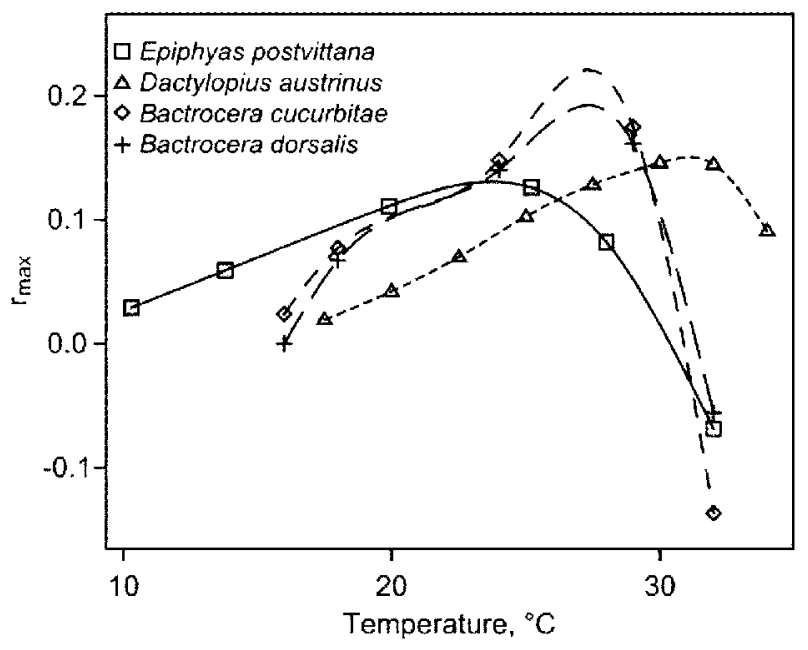

Figure 1: Plots of $r_{\max }(1 / \mathrm{d})$ as a function of $T\left({ }^{\circ} \mathrm{C}\right)$ for four species of insects. This is an example of a typical temperature profile of $r_{\max }$. We took the optimal temperature to be $25.2^{\circ} \mathrm{C}$ for Epiphyas postvittana, $29^{\circ} \mathrm{C}$ for Bactrocera dorsalis, $29^{\circ} \mathrm{C}$ for Bactrocera cucurbitae, and $32^{\circ} \mathrm{C}$ for Dactylopius austrinus. We also excluded the data for $B$. dorsalis at $16^{\circ} \mathrm{C}$ because the population growth rate was negative. This figure illustrates the "biologically relevant" temperature range for these four species and is representative of plots for the other species used in this article.

dependence. Outside of this biologically relevant range, factors such as the freezing of water at low temperatures or the breakdown of proteins at high temperatures have negative effects on metabolism and population growth. As seen in figure 1 , the optimal temperature is $25.2^{\circ} \mathrm{C}$ for Epiphyas postvittana, $29^{\circ} \mathrm{C}$ for Bactrocera dorsalis and Bactrocera cucurbitae, and $32^{\circ} \mathrm{C}$ for Dactylopius austrinus. While understanding effects of temperature outside of the biologically relevant range is an interesting problem, it is beyond the scope of this article. Here, in compiling and analyzing data, we have excluded temperatures that are clearly below the minimum threshold for positive population growth and definitively above the optimal temperature, that is, low temperatures with nonpositive population growth rates and high temperatures where population growth rates have begun to decline dramatically. For example, we excluded the data for $B$. dorsalis at $16^{\circ} \mathrm{C}$ because the population growth rate was negative. Figure 1 contains other examples of data that were excluded for being outside the biologically relevant temperature range.

Data for insects from the article by Banse and Mosher (1980) demonstrated a mass dependence $\left(r_{\max } \propto M^{1.21}\right)$ that was anomalous, with a positive allometric exponent for $r_{\max }$ compared with the negative exponents $(\sim-0.25)$ found in insect data from all other sources. We believe this to be in error, so these data were excluded from our data set.

The data include three species of algae, one species of protist, eight species of zooplankton, and five species of insects. Body masses of some species were not mentioned in the published articles, so values for some zooplankton in appendix B in the online edition of the American Naturalist were taken from those of Gillooly (2000). When there were multiple data points for the same species at the same temperature, we computed an average value to avoid a biased weighting for those species and temperatures. For details of methods, see the data sources referenced in the supplemental "Literature Cited" in the online edition of the American Naturalist.

The data for all groups were then logarithmically transformed, plotted, and fit with Type I linear regression. Type I regression is most appropriate because mass and temperature are measured with a high degree of precision while population growth rates often are not.

\section{$r_{\max }$ and $Z$ : Laboratory and Field Data for Fishes and Mammals}

The data for intrinsic rate of increase, $r_{\max }$, for fishes were compiled from estimates from field studies. Data for mammals were derived from direct measures in laboratory studies. The data for mammals are for only four species, all rodents. Because these closely related taxa are the only data for high temperatures, we address concerns about nonindependence as a result of phylogenetic relatedness by also calculating regression lines with the mammal data excluded. The body temperature for mammals is approximately constant $\left(\sim 37^{\circ} \mathrm{C}\right)$. The temperature for fishes was taken as the average temperature at which they live. Because most of the data is for marine fishes, there is little seasonal variation, so using the average temperature in equation (8) is a good approximation. In the original sources, data are given in terms of production per unit biomass, which is equal to $r_{\max }$ for populations with a stable body size distribution. The data include nine species of fishes and four species of mammals. Data for mortality rates, $Z$, for marine fishes (175 stocks) were taken from a compilation by Pauly (1980). Methods are detailed in the references in the supplemental "Literature Cited." The data for all groups were combined, logarithmically transformed, plotted, and fit with Type I linear regression.

\section{Results}

Before analyzing different taxonomic groups, we did an ANOVA on all of the $r_{\max }$ data. The ANOVA gives values for independent regressions of $r_{\max }$ as a function of $1 / k T$ and as a function of $\ln (M)$. Further, it determines whether 
the effects of these two variables are independent. Using the data for $r_{\max }$, we obtain an activation energy of 0.63 $\mathrm{eV}(P<.01 ; 95 \%$ confidence interval [CI]: $0.52,0.74)$ and a scaling exponent for the mass of $-0.22(P<.01 ; 95 \%$ CI: $-0.20,-0.23)$. The value for the activation energy is in close agreement with the value of $0.6 \mathrm{eV}$ for the average of the activation energies for cellular biochemical reactions (range, 0.2-1.2 eV; Crossier 1926; Raven and Geider 1988; Vetter 1995; Gillooly et al. 2001). The value for the mass exponent is close to but slightly lower than the predicted exponent, -0.25 . This deviation from theoretical explanation may be a result of the slightly different intercepts among taxonomic groups.

As indicated, the $M^{-1 / 4}$ mass dependence of $r_{\max }$ is well documented (Fenchel 1973; Southwood et al. 1974; Blueweiss et al. 1978; Henneman 1983; Peters 1983), so we focused primarily on evaluating the temperature dependence of $r_{\max }$ predicted by equation (8). Because the activation energy, $E$, varies little across taxa and equation (8) is a good approximation for populations described by case 1 , we predict that plots of $\ln \left(r_{\max } M^{1 / 4}\right)$ versus $1 / k T$ will yield straight lines with an approximately invariant slope, $-E$, and $y$-intercept, $\ln \{\ln [L(G) b(G)] / C\}$. We also predict the negative of the slopes $(E)$ should be close to the value of $0.6 \mathrm{eV}$ for the average activation energies for cellular and biochemical reactions (Crossier 1926; Raven and Geider 1988; Vetter 1995; Gillooly et al. 2001). We first evaluate this prediction using empirical measurements of $r_{\max }$ from two groups of unicellular organisms with nonoverlapping generations grown at different constant temperatures in the laboratory, protists and algae. As shown in figure 2, left panel, plots of $\ln \left(r_{\max } M^{1 / 4}\right)$ versus $1 / k T$ do indeed yield straight lines. The slope of the line for these unicellular eukaryotes is $-0.54 \mathrm{eV}(P<.01 ; 95 \%$ CI:
$-0.40,-0.68 \mathrm{eV})$. Plotting the two groups separately gives a slope for algae of $-0.53 \mathrm{eV}(P<.01 ; 95 \%$ CI: -0.32 , $-0.75 \mathrm{eV})$ and for the protists, Paraphysomonas imperforata, $-0.65 \mathrm{eV}(P<.01 ; 95 \% \mathrm{CI}:-0.41,-0.89 \mathrm{eV})$. All of these plots and regression lines for individual species have slopes and confidence intervals that are within the range of activation energies for cellular biochemical reactions. Regressions lines were also fitted to the data for each species, and the slopes are all within the range of activation energies for cellular biochemical reactions.

Data for multicellular organisms with nonoverlapping generations, zooplankton (rotifers, copepods, and cladocerans) and insects (mostly agricultural pests), also support the hypothesis for case 1 , that $r_{\max }$ scales inversely with biological time. A plot for these groups yields a straight line with slope $-0.84 \mathrm{eV}(P<.01 ; 95 \% \mathrm{CI}:-0.65,-1.03$ eV; fig. 2, center panel). Plotting the two groups separately gives slopes of $-0.85 \mathrm{eV}(P<.01 ; 95 \% \mathrm{CI}:-0.56,-1.14$ $\mathrm{eV})$ for zooplankton and $-0.53 \mathrm{eV}(P<.01 ; 95 \% \mathrm{CI}$ : $-0.28,-0.79 \mathrm{eV})$ for insects. Regression lines were also fitted to the data for each species, and the slopes are all within the range of cellular biochemical reactions.

The hypothesis that the scaling for $r_{\max }$ also applies to case 2 , and the possible extension of case 2 to indeterminate ontogenetic growth, is supported by data for multicellular organisms with overlapping generations, fishes. The solid line (fit to the fish data) shown in figure 2, right panel, is a straight line with a slope of $-0.35 \mathrm{eV}(P=$ .05 ; $95 \%$ CI: $0.00,-0.69 \mathrm{eV})$. These values for $E$ are lower than most of the activation energies for cellular biochemical reactions (Crossier 1926; Raven and Geider 1988; Vetter 1995; Gillooly et al. 2001). However, these values are in agreement with the activation energy for fishes of -0.43 $\mathrm{eV}$, which was derived from data for metabolic rate (Gil-
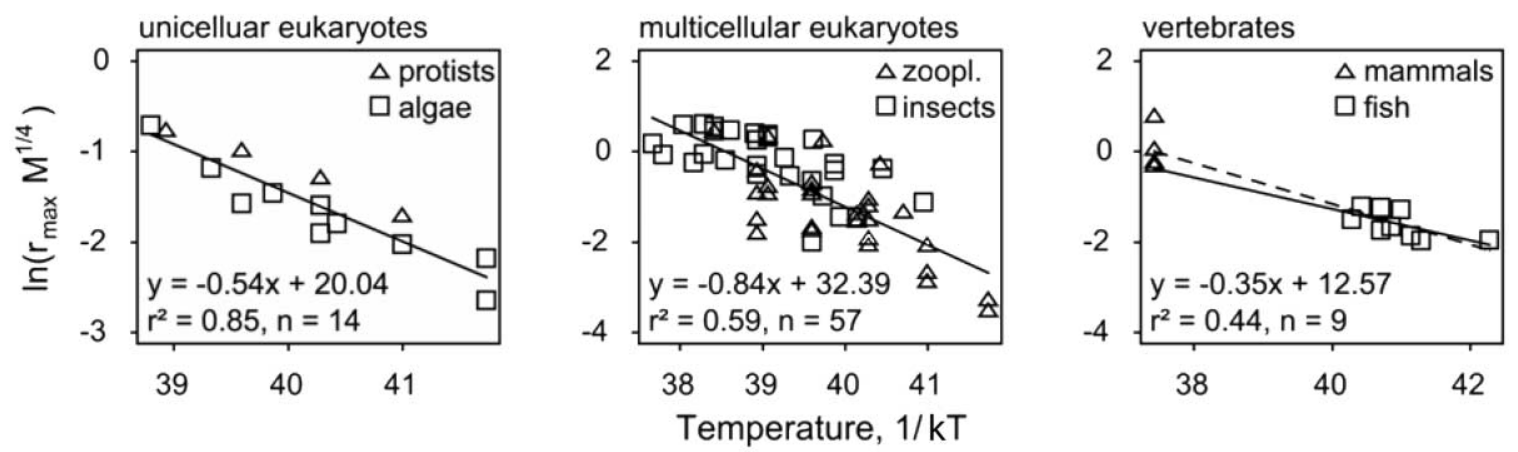

Figure 2: Relationship of mass-corrected exponential population growth, $r_{\max }$ (individuals/[individual $\times$ day]) $\times M^{1 / 4}\left(\right.$ fresh weight, $\left.\mu \mathrm{g}^{1 / 4}\right)$, to inverse temperature, $T(1 / K)$, for unicellular invertebrates (left panel, algae and protists) and multicellular invertebrates (center panel, zooplankton and insects) that were incubated at different constant temperatures in the laboratory. The same type of plot is performed using data for fishes (right panel, solid line) and fishes combined with mammals (dashed line). Lines were fit using Type I regression. The equation is for the fit to the fish data only. The data were obtained from published sources listed in the supplemental "Literature Cited." As predicted, the plots are straight lines. 
looly et al. 2001). Further, Gillooly et al. (2001) showed that after accounting for mass and temperature, the variation in metabolic rate between most ectotherms and endotherms reduces to a factor of six. Because we have related population growth rates to metabolic rates, it seems that a similar offset may exist between the population growth rates of fishes and mammals. However, combining data for mammals and fishes, we find that for population growth rates, the difference is reduced to an even smaller factor. The dashed line (fit to fish and mammal data) shown in figure 2, right panel, is a straight line with a slope of $-0.45 \mathrm{eV}(P<.01 ; 95 \% \mathrm{CI}:-0.33,-0.57 \mathrm{eV})$. Because the fit to both fish and mammal data spans a broader range of temperatures, the fit to the combined data may be a more accurate determination of the activation energy for these two groups.

To determine whether $Z$ follows equation (16) for organisms with overlapping generations and indeterminate ontogenetic growth, we use estimates of instantaneous mortality rate for a broad assortment of marine fishes (175 stocks) living under field environmental conditions. If $Z$ follows equation (16), we predict once again that a plot of $\ln \left(Z M^{1 / 4}\right)$ versus $1 / k T$ will yield a straight line. Figure $3 A$ supports this prediction with a straight line and a slope of $-0.45 \mathrm{eV}(P<.01 ; 95 \% \mathrm{CI}:-0.36,-0.53 \mathrm{eV})$. This again is within the range of activation energies for cellular biochemical reactions and is in close agreement with the activation energy for fish of $-0.43 \mathrm{eV}$ (Gillooly et al. 2001). As a further test of this prediction, equation (16) predicts that a plot of $\ln \left(Z e^{E / k T}\right)$ versus $\ln (M)$ will yield a straight line with a slope of -0.25 . When we use $0.45 \mathrm{eV}$ for the activation energy, as measured from the previous graph, figure $3 B$ strongly supports this prediction with a straight line and a slope of $-0.23(P<.01 ; 95 \% \mathrm{CI}:-0.20,-0.27)$.

The temperature dependence of mortality is of great importance in understanding how fish mortality rates differ between ecosystems. A negative quarter-power mass scaling for mortality was also found empirically by Peterson and Wroblewski (1984). McGurk (1986) went even further and developed a model based on spatial patchiness that describes the deviations from this scaling, which apply primarily to fish eggs and larvae. Peppin (1991) studied the combined effects of body mass and temperature on mortality rates for early life-history stages of marine fishes. Because our data are for marine fishes in the field, figure 3 indicates that the natural forces causing mortality (e.g., predation) scale predictably with the rate of metabolism. That is, an organism's ability to avoid death appears to diminish with age at a rate set by the mass-specific metabolic rate. Therefore, survivorship, which depends largely on an organism's ability to acquire food and evade predators, should scale inversely with mass-specific metabolic rate, $\bar{B}(M, T)$, as observed in figure 3. Equation (16) also predicts that $L(\alpha)=e^{-\bar{Z} \alpha}$ is an invariant, independent of body mass and temperature. Consequently, for diverse organisms living in systems of differing temperatures, we predict that approximately the same percentage of offspring will survive to the age of first reproduction.

The data sets for algae and protists, insects and zooplankton, and vertebrates support the predictions of the theory relating population growth characteristics to metabolic rate and thereby to body size and temperature. Gillooly et al. (2001) found that the metabolic rates of diverse eukaryotes had quantitatively similar mass and temperature dependence, so after mass and temperature correction, the rates overlapped substantially among different taxonomic groups. This suggests that once the data for $r_{\max }$ have been corrected for mass and temperature, they might also exhibit considerable overlap. Figure $4 \mathrm{~A}$ evaluates this by plotting mass-corrected $r_{\max }$ as a function of
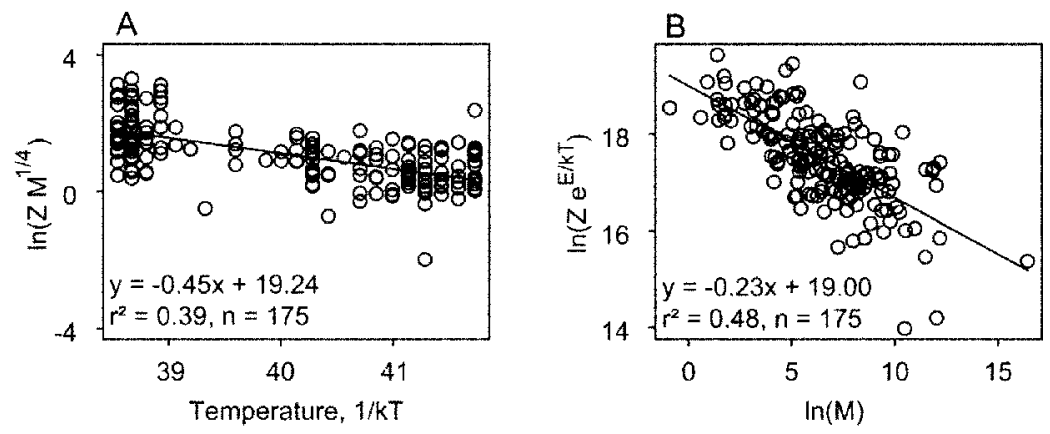

Figure 3: $A$, Relationship of the mass-corrected instantaneous mortality rate, $Z$ (individual/[individual $\times$ years]) $\times M^{1 / 4}\left(\mathrm{~g}^{1 / 4}\right)$, to inverse temperature, $T(1 / K)$, for marine fishes. $B$, Relationship of the temperature-corrected instantaneous mortality rate, $Z e^{E / k T}$ (individual/[individual $\times$ years]), to body mass, $M(\mathrm{~g})$, for marine fishes. On the basis of $A$, an activation energy of $0.45 \mathrm{eV}$ was chosen. The lines were fit using Type I linear regression. The data were obtained from a single reference listed in appendix B. Both plots give straight lines, as predicted. 

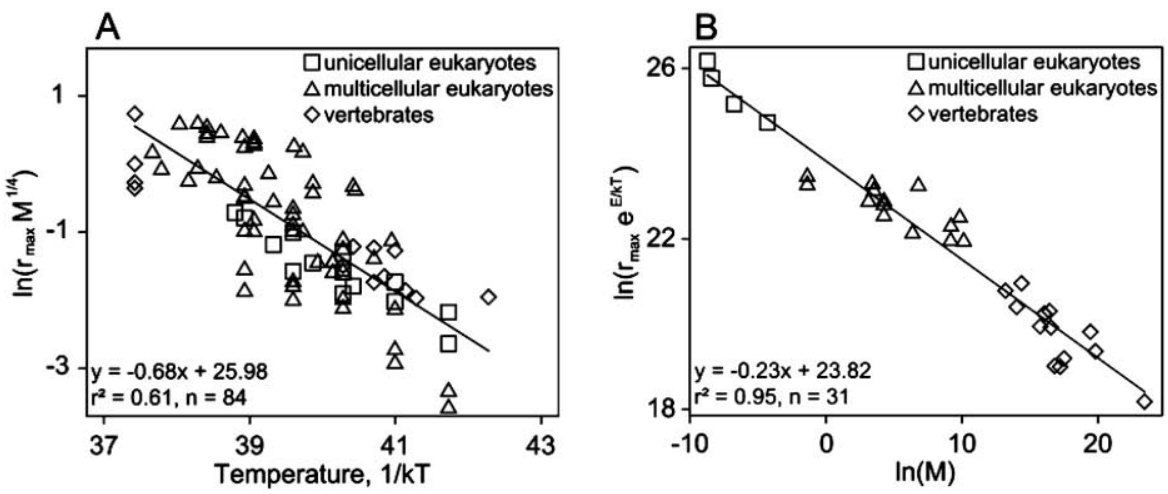

Figure 4: $A$, Relationship of mass-corrected exponential population growth, $r_{\max }$ (individuals/[individual $\times$ day]) $\times M^{1 / 4}\left(\right.$ fresh weight, $\left.\mu \mathrm{g}^{1 / 4}\right)$, to inverse temperature, $T(1 / K)$, for all organisms in figure 2 . There is significant overlap among the data, and a single line is fit through the data. $B$, Relationship of temperature-corrected exponential population growth, $r_{\max } e^{E / k T}$ (individuals/[individual $\times$ day]), to body mass, $M(\mu \mathrm{g})$, for all organisms in figure 2. $E$ was chosen to be $0.63 \mathrm{eV}$ on the basis of an ANOVA, and each point represents the average of the logarithm of temperaturecorrected $r_{\max }$ for each species. The line was then fit using Type I linear regression. All of the data are well fit by this single straight line.

inverse temperature, using a mass exponent of $1 / 4$. These data span 12 orders of magnitude of variation in body size. Figure $4 A$ shows not only the similar activation energies (slopes) but also the overlapping values of $r_{\max }$ across a wide variety of organisms, from unicells to mammals. The nearly complete overlap across organisms with diverse modes of reproduction (i.e., fission, ovipary, and vivipary) implies that population growth rates have very nearly the same mass and temperature dependence. Figure $4 B$ is a plot of temperature-corrected $r_{\max }$ as a function of body size. To prevent an unbiased weighting of the data, we have taken the average of the logarithm of the temperature-corrected $r_{\max }$ for each species, so each point in figure $4 B$ represents a single species. Also, an activation energy of $0.63 \mathrm{eV}$ was chosen on the basis of the ANOVA discussed in the first paragraph of this section. Note that the fitted regression line now gives a slope of -0.23 for the mass exponent, slightly higher than the ANOVA value of -0.22 . This is due to the effect of averaging over temperature for each species. Here, we see that across 12 orders of magnitude of variation in body size, a single line describes the mass dependence of $r_{\max }$ for the broad assortment of organisms studied in this article.

\section{Discussion}

This theory characterizes the fundamental effects of body size and temperature. When the predictions are evaluated empirically, we see that most of the variation in rates of population growth is explained by this mass and temperature dependence, $M^{1 / 4} e^{-E / k T}$. The activation energies derived from population growth data for the taxonomic groups considered here vary from 0.35 to $0.84 \mathrm{eV}$. These bracket the average value for the activation energies of metabolic reactions, $0.6 \mathrm{eV}$, and the average value obtained from metabolic range data, $0.62 \mathrm{eV}$ (Gillooly et al. 2001), although the causes of this variation are not clear. The question of whether there is a unique activation energy that determines how metabolic rate scales with temperature remains open.

These scaling relations, which hold over 12 orders of magnitude in body mass and over approximately $30^{\circ} \mathrm{C}$, emphasize the commonality of life. This unity is expressed in the dependence of population growth rate and carrying capacity on metabolic rate. Presumably, population growth is so tightly linked to metabolism because all organisms use the same fundamental metabolic reactions for energy transformation and biosynthesis (Morowitz 1992). The mass dependence of biosynthesis reflects the fractal-like design and resulting rate-limiting constraints of the resource supply system. The temperature dependence of biosynthesis reflects the kinetics of the metabolic reactions.

The data do not always match the theoretical predictions, and these differences may have significant biological meaning. Nevertheless, much of the variation in rates of population growth can be explained solely by the mass and temperature dependence. Thus, this theory can now be used as a baseline or zero-order model to explore the remaining variations that may reveal important biological insights. Understanding the commonality for this broad assortment of eukaryotic organisms may help to identify and better understand how and why some species are different. The direction and magnitudes of deviations from predicted slopes or fitted regression lines offer clues to the identity of other important variables and the magnitudes and mechanisms of their influence. In some cases, depar- 
tures may call attention to errors or biases due to experimental or measurement techniques or to discrepant results due to different methodologies. Other departures may reflect important differences between different kinds of organisms or environments. For example, there is considerable variation in size-corrected ontogenetic growth rates of zooplankton around the regression lines for temperature. Much of this residual variation can be attributed to growth rates of individuals and species increasing with body phosphorus concentrations (Gillooly et al. 2002). This is just what is predicted by the growth rate hypothesis of Elser et al. (1996; Sterner and Elser 2002). We expect many other sources of biological variation to be identified in a similar manner.

We recognize that the models developed here and in related publications (Gillooly et al. 2001, 2002; Allen et al. 2002; Brown et al. 2003; Jun et al. 2003; Moses and Brown 2003) neither explain all of the empirically observed variation nor provide a complete mechanistic explanation for the factors and processes controlling population growth. We view these models as preliminary contributions to a more complete metabolic theory of ecology. When complete, such a theory would make explicit the environmental and physiological factors controlling the fluxes and transformations of energetic and material resources at the individual level: uptake from the environment; the biochemical and physiological processing within the body; and allocation to survival, growth, and reproduction. To do this, the theory would explicitly incorporate ecological stoichiometry (Elser et al. 1996; Sterner and Elser 2002) and would include more detailed studies of mass and energy relationships at the cellular, individual, and population levels (e.g., Kooijman et al. 1991). It would also incorporate the long-standing research program in microbiology and entomology that has related resourcelimited population growth to underlying biochemical processes (e.g., Monod 1942; Birch 1948; Dean and Hinshelwood 1966; Droop 1968; Eppley 1972). Developing a comprehensive metabolic theory of ecology would connect many seemingly disparate parts of ecology and, hence, help to unify the field. Much remains to be done before there will be any reasonably complete metabolic theory of ecology. Nevertheless, the progress has been encouraging, and the prospects are exciting.

In this article, we have linked population growth to metabolism and thereby to mass and temperature. This already has several important implications for ecology. First, it relates two different types of population growth, Malthusian population growth and its fundamental parameter, $r_{\max }$, and steady state population growth and its fundamental parameter, $K$, to first principles of energetics and kinetics. It links the one unquestioned law of population ecology to underlying mechanistic laws of physics and physical chemistry. Second, this theory extends the theoretical framework initiated by Charnov (1993) to make connections between metabolism (production) and features of life history, such as ontogenetic growth rate, timing and magnitudes of fecundity and mortality, and life span. Third, this theory begins to make explicit how metabolic processes at the level of individual organisms affect ecological processes by determining the magnitudes, rates, and times of impacts on and responses to environments.

Additionally, it should be possible to extend this framework to address other important patterns and processes in ecology and evolution. Metabolism links the requirements and transformations of energy and materials in the bodies of organisms to the biotic and abiotic pools and fluxes of energy and materials in the environment. Theoretical explorations of these linkages are already offering new insights into such diverse phenomena as the structure and dynamics of forests (Enquist et al. 1998; Enquist and Niklas 2001, 2002), foraging behavior of ants (Jun et al. 2003), latitudinal and elevational patterns of species diversity (Allen et al. 2002), geographic variation in the diversity of aboriginal human cultures (Collard and Foley 2002), and fecundity rates in modern human populations (Moses and Brown 2003).

\section{Acknowledgments}

We thank D. Faguy, A. Hammond, and P. Turchin for helpful discussions. We thank D. Allen for helpful discussions and for help making figures. V.M.S. is grateful for the support of the National Science Foundation (NSF) and E.L.C. for the support received as a MacArthur Fellow. J.F.G., G.B.W., and J.H.B. are grateful for the support of the Thaw Charitable Trust, a Packard Interdisciplinary Science Grant, and an NSF Biocomplexity Grant. This research is also supported by the U.S. Department of Energy, under contract W-7405-ENG-36.

\section{Literature Cited}

Allen, A. P., J. F. Gillooly, and J. H. Brown. 2002. Global biodiversity, biochemical kinetics, and the energeticequivalence rule. Science 297:1545-1548.

Banavar, J. R., A. Maritan, and A. Rinaldo. 1999. Size and form in efficient transportation networks. Nature 399: 130-132.

Banavar, J. R., J. Damuth, A. Maritan, and A. Rinaldo. 2002. Supply-demand balance and metabolic scaling. Proceedings of the National Academy of Sciences of the USA 99:10506-10509.

Banse, K., and S. Mosher. 1980. Adult body mass and annual production/biomass relationships of field populations. Ecological Monographs 50:355-379. 
Belgrano, A., A. P. Allen, B. J. Enquist, and J. F. Gillooly. 2002. Allometric scaling of maximum population density: a common rule for marine phytoplankton and terrestrial plants. Ecology Letters 5:611-613.

Birch, L. 1948. The intrinsic rate of increase of an insect population. Journal of Animal Ecology 17:15-26.

Blueweiss, L., H. Fox, V. Kudzma, D. Nakashima, R. Peters, and S. Sams. 1978. Relationships between body size and some life history parameters. Oecologia (Berlin) 37:257272.

Brown, J. H., J. F. Gillooly, G. B. West, and V. M. Savage. 2004. Toward a metabolic theory of ecology. Ecology (in press).

Charnov, E. L. 1993. Life history invariants. Oxford University Press, Oxford.

Collard, I. F., and R. A. Foley. 2002. Latitudinal patterns and environmental determinants of recent human cultural diversity: do humans follow biogeographical rules? Evolutionary Ecology Research 4:371-383.

Crossier, W. J. 1926. The distribution of temperature characteristics for biological processes: critical increments for heart rates. Journal of General Physiology 7:137149.

Damuth, J. 1987. Interspecific allometry of population density in mammals and other animals: the independence of body mass and population energy use. Biological Journal of the Linnaean Society 31:193-246.

Dean, A. C. R., and C. N. Hinshelwood. 1966. Growth, function and regulation in bacterial cells. Clarendon, Oxford.

Droop, M. R. 1968. Vitamin B12 and marine ecology. IV. The kinetics of uptake, growth, and inhibition in Monochrysis lutheri. Journal of the Marine Biological Association of the UK 48:689-733.

Elser, J. J., D. Dobberfuhl, N. A. Mackay, and J. H. Schampel. 1996. Organism size, life history and N : P stoichiometry: toward a unified view of cellular and ecosystem processes. BioScience 46:674-684.

Enquist, B. J., and K. J. Niklas. 2001. Invariant scaling relations across tree-dominated communities. Nature 410:655-660.

- 2002. Global allocation rules for patterns of biomass partitioning in seed plants. Science 295:1517-1520.

Enquist, B. J., J. H. Brown, and G. B. West. 1998. Allometric scaling of plant energetics and population density. Nature 395:163-165.

Eppley, R. W. 1972. Temperature and phytoplankton growth in the sea. Fisheries Bulletin 70:1063-1085.

Fenchel, T. 1973. Intrinsic rate of natural increase: the relationship with body size. Oecologia (Berlin) 14:317326.

Gillooly, J. F. 2000. Effect of body size and temperature on generation time in zooplankton. Journal of Plankton Research 22:241-251.

Gillooly, J. F., J. H. Brown, G. B. West, V. M. Savage, and E. L. Charnov. 2001. Effects of size and temperature on metabolic rate. Science 293:2248-2251.

Gillooly, J. F., E. L. Charnov, G. B. West, V. M. Savage, and J. H. Brown. 2002. Effects of size and temperature on developmental time. Nature 417:70-73.

Hennemann, W. W., III. 1983. Relationships among body mass, metabolic rate, and the intrinsic rate of natural increase in mammals. Oecologia (Berlin) 56:104-108.

Jun, J., J. W. Pepper, V. M. Savage, J. F. Gillooly, and J. H. Brown. 2003. Allometric scaling of ant foraging trail networks. Evolutionary Ecology Research 5:297-303.

Kooijman, S. A. L. M., E. B. Muller, and A. H. Stouthamer. 1991. Microbial growth dynamics on the basis of individual budgets. Antonie von Leeuwenhoek 60:159174.

Malthus, T. 1798. An essay on the principle of population. Macmillian, New York.

May, R. M. 1976. Estimating $r$ : a pedagogical note. American Naturalist 110:496-499.

McGurk, M. D. 1986. Natural mortality of marine pelagic fish eggs and larvae: role of spatial patchiness. Marine Ecology Progress Series 34:227-242.

Monod, J. 1942. Recherches sur la croissance des cultures bacteriennes. Hermann, Paris.

Morowitz, H. J. 1992. Beginnings of cellular life: metabolism recapitulates biogenesis. Yale University Press, New Haven, Conn.

Moses, M., and J. H. Brown. 2003. Allometry of human fertility and energy use. Ecology Letters 6:1-6.

Pauly, D. 1980. On the interrelationships between natural mortality, growth parameters, and mean environmental temperature in 175 fish stocks. Journal du Conseil Conseil International pour l'Exploration de la Mer 39:175192.

Peppin, P. 1991. Effect of temperature and size on development, mortality, and survival rates of the pelagic early life history stages of marine fish. Canadian Journal of Fisheries and Aquatic Sciences 48:503-518.

Peters, R. H. 1983. The ecological implications of body size. Cambridge University Press, Cambridge.

Peterson, I., and J. S. Wroblewski. 1984. Mortality rates of fishes in the pelagic ecosystem. Canadian Journal of Fisheries and Aquatic Sciences 41:1117-1120.

Raven, J. A., and R. J. Geider. 1988. Temperature and algal growth. New Phytologist 110:441-461.

Savage, V. M. 2004. Improved approximations to scaling relationships for species, populations, and ecosystems across latitudinal and elevational gradients. Journal of Theoretical Biology (in press).

Southwood, T., R. May, M. Hassell, and G. Conway. 1974. 
Ecological strategies and population parameters. American Naturalist 108:791-804.

Sterner, R. W., and J. J. Elser. 2002. Ecological stoichiometry: the biology of elements from molecules to the biosphere. Princeton University Press, Princeton, N.J.

Turchin, P. 2001. Does population ecology have general laws? Oikos 94:17-26.

Vetter, R. A. H. 1995. Ecophysiological studies on citrate- synthase. I. Enzyme regulation of selected crustaceans with regard to temperature adaptation. Journal of Comparative Physiology B 165:46-55.

West, G. B., J. H. Brown, and B. J. Enquist. 1997. A general model for the origin of allometric scaling laws in biology. Science 276:122-126.

Associate Editor: Jonathan B. Losos 